\title{
OBJECT-BASED IMAGE ANALYSIS BEYOND REMOTE SENSING - THE HUMAN PERSPECTIVE
}

\author{
T. Blaschke ${ }^{\mathrm{a}, *}$, S. Lang ${ }^{\mathrm{a}}$, D. Tiede ${ }^{\mathrm{a}}$, M. Papadakis ${ }^{\text {a }}$, A. Györi ${ }^{\mathrm{a}}$ \\ a Dept. of Geoinformatics, University of Salzburg, Schillerstr. 30, 5020 Salzburg, Austria (thomas.blaschke, stefan.lang, dirk.tiede, \\ emmanouil.papadakis, anna.gyori )@sbg.ac.at
}

Thematic Session 15

KEY WORDS: OBIA, GEOBIA, GIScience, place, bona fide objects

\begin{abstract}
:
We introduce a prototypical methodological framework for a place-based GIS-RS system for the spatial delineation of place while incorporating spatial analysis and mapping techniques using methods from different fields such as environmental psychology, geography, and computer science. The methodological lynchpin for this to happen - when aiming to delineate place in terms of objects - is object-based image analysis (OBIA).
\end{abstract}

\section{INTRODUCTION}

Remote Sensing imagery has literally arrived at everybody’s desktop. The increasing availability and accessibility along with improving resolutions created interests among various domain experts - many of them not having remote sensing backgrounds. One may claim that the synoptic view triggered this popularity. However, increasing amounts of data do not necessarily nor automatically lead to tangible information. This quest for tangible information favoured the marriage of GIStechniques and image processing and the fast developing paradigm of object-based image analysis (OBIA, see Blaschke 2010), often referred to as Geographic object-based image analysis (GEOBIA) when distinguishing applications from other domains such as medical imaging or material sciences which also utilize OBIA methods (Blaschke et al. 2014).

In this synthesis paper we briefly highlight the merger of GIS and remote sensing through OBIA with an emphasis to address human-centric perceptions - rather than geometric (sub-)entities directly visible in images. Like any computer software and method GIS is limited by the type and amount of data that are available. GIS often contains dozens of layers of data. Nevertheless, the ever increasing volume of data frequently masks the fact that qualitative data, which has great relevance in the human beings behind the digital numbers, is missing. Several qualitative GIS approaches - predominantly in North America - point out how GIS could equally capable of storing and representing both qualitative and quantitative data. Qualitative GIS maybe an important component when trying to understand what a location means to humans. Place is an increasingly important notion in geographic information science (GIScience) - although the place-based literature accounts for a tiny fraction of the overall GIS literature. Places are understood as locations that are made meaningful by people who become attached to them. In general, it is difficult to analyse such qualitative data on a computer. Unfortunately, except for an initiative in the late 1990s ("socializing the pixel") such a human-centric consideration is basically absent in remote sensing sciences.
GIS has arrived at everybody's desktop. So do remote sensing images - if we include services like Google Maps, Google Earth or Microsoft Bing maps (Blaschke et al. 2012). While it is believed that a million or so people across the globe work daily with GIS software, certainly more than one hundred million users use widely accessible mapping sites - such as Google Maps. GIS functions are increasingly embedded into such services while at the same time we are witnessing an increasing amount of complexity in applications.

Several examples illustrate solutions to go beyond the notion of GIS as a managing and analysing system that provides spatial facts on the one hand side and remote sensing imagery as being limited to the 'roof-top view' on the other. Addressing an human-centred view the examples underpin the importance of facts as well as opinions, emotions and feelings surrounding such spatial facts. We will introduce a prototypical methodological framework and preliminary solutions for a place-based GIS-RS system for the spatial delineation of place while incorporating spatial analysis and mapping techniques using innovative methods from different fields such as environmental psychology, geography, and computer science. The methodological lynchpin for this to happen - when aiming to delineate place in terms of objects - is OBIA. It seems to be obvious that such objects are fiat objects but they may relate to bona fide objects such as streets, buildings, or trees. One overarching research question which is tackled by two aligned $\mathrm{PhD}$ theses is therefore do such place-based objects differ from conventional spatial objects also in terms of their outline and, subsequently, in terms of shape and size?

\section{PLACE AND OBJECTS}

There are various representation techniques for visualizing, describing and analyzing the geographical world. "From puzzles of polygons to sandwiches of data layers" (Couclelis, 1992), GIS utilizes geometry, statistics, maths and physics in order to provide a precise and scientifically-defined view of space. However, is it possible for a set of geometrical features

* Corresponding author 
along with some grid cell values to reflect the geographical world as it is projected in the human mind?

People are familiar with space in a scientific point of view; however they do not intuitively refer to any kind of raster or vector representations when they are using spatial references. It would be valid to claim that GIS space's representation is bounded to the mathematical and physical description of space. On the contrary, space, as perceived and conceived by people hereinafter place, moves beyond spatial descriptions to semantic definitions of space.

Relph (1992) construes place as a means for people to integrate human intentions, experiences and actions in the spatial dimension. The association of physical space and place is portrayed by Tuan (1977) as follows: "place is space infused with human meaning". Integration of place in GIS platforms reveals an attempt to bridge the human meaning of space with its underlying spatial projection. Such an accomplishment would operationalize what Curry [4] defined as place, that is, a description of physical space in a human-driven way.

If a concept of place-based GIS-RS is to be realized, the incorporated scientific components, that is, GIS and Remote Sensing, should keep pace with the human ways. People perceive space in a qualitative way (Andrew, 1999) and they describe it qualitatively, as well. They introduce places in the sense of naming, categorizing, symbolizing, narrating or associating space (Curry, 1991). The importance of qualities instead of quantities is indisputable; for instance, a placename is better conceived as a spatial location rather than a set of coordinates. Focusing on more complicated GIS methods, the introduction of spatial relations (Egenhofer \& Franzosa, 1991), such as overlaps or touches, conforms better to the human understanding of space, rather than a percentage of coverage or buffered representations of vicinity, respectively.

In the plane of remote sensing, qualitative data can be considered similarly as the indicators used in the field of Quality of Life. Particularly, qualities assign a human meaning, or better stated, a symbol to the quantitative values that describe space. For instance, the vegetation indicator value of 0.4 does not reflect the human intuition as accurately as the nominal value of "green area" does. Consequently, remote sensing can support the definition of place by converting and grouping sensor data into categorical values, constraining their numerical part exclusively in system calculations and algorithms.

Moving to a more abstract understanding of the world, humans tend to think with objects (Couclelis, 1992). It is an intuitive process of breaking down a complex system, such as the real world, into simpler, (partly) semantically independent and meaningful wholes, which are easier to describe e.g. recognizing a mountain out of a forestry area. These sophisticated entities are annotated with properties and associations that enable a composite view of the world, in the sense of interconnected components that build the whole world.

With respect to place-based GIS, the introduction of geographic entities can also support the human understanding of space. Geographical entities can range from simple objects, either physical or conceptual, such as a tree or a parking slot, to composite heterogeneous elements such as a forest, a parking lot, a town or any place, in general. Considering place as a composite object uplifts the integrated projection of space from a "brute spatial existence" to a sophisticated entity with properties and associations, which are derived from its constituent spatial components. For instance, a park is green because it is composed of trees and grassy areas.

A leading method supporting this approach is OBIA (Blaschke, 2010), which partitions remote sensing data into meaningful objects. Apart from the segmentation process of OBIA, the classification process is of high importance for describing place. Simple components with similar context, geometry, structure and texture can be grouped into certain classes. The assignment of semantic and spatial properties to the simplest categories of objects can, in turn, facilitate the definition of properties for objects of higher complexity, which are composed of the simpler ones. Figure 1 illustrates an example of water garden composition from grassy and water patches in Mirabell gardens in the city of Salzburg. Consequently, this enables a bottom-up description of place constructed by a recursive process that moves from simpler to composite objects.

\section{APPLICATION: QUALITY OF LIFE}

As a possible application example for this human-centred place concept can be the analysis of the Human Scale and the linkage of it to the Quality of Life research which has not been explicitly happened so far. The Human Scale concept was developed by a Danish architect, Jan Gehl. He pointed out that the urban planning and architecture paradigm shifted into a harmful direction when the architects and engineers started to plan cities from "helicopter perspective" and design everything for cars which had significantly higher speed and different needs for place than the pedestrians (Gehl, 2010). Therefore, the interest of citizens who intended to use the public spaces without a car got neglected and so the cities became lifeless and neutral. However, through the analysis of the senses and perception of the human beings and applying it for urban planning the public life can be brought back to cities (Gehl, 1987, Gehl, 2010, Gehl \& Svarre, 2013). The concept of Human Scale is widely used within urban planning and architecture considered as a rather qualitative attribute or a desirable state to describe a liveable city. As a new approach, its quantitative aspects could also get more emphasis by applying GIS methods to become more transferable and tangible. For this purpose, a set of predefined quantitative factors are needed since for a computer application everything should be measurable, comparable and reproducible. This introduces and requires the importance of the combination of at least three different concepts: the human perception, the remote sensing, and the application of GIS.

The other - currently being developed - practical use case where the new aspect from the human perspective together with the utilization of GIS is present is the algorithm for creating meaningful place units. The main concept behind it relates to the definition of neighbourhoods and the difficulties of defining it. Despite being a fundamental unit in many disciplines there is no explicit definition due to the various meanings it may have with differing cultural and historical backgrounds. This diverse interpretation introduces challenges especially while delineating them with GIS methods because there is a need for general and transferable rules. As a first step of segmenting a city using human perception of space, the purpose of the concept was to create these "meaningful place units" based on physical and 
psychological barriers, and proximity dependent on accessibility and context. To assess how strongly these physical features can act as a barrier from the human perspective weights were applied - specified according to psychological expert knowledge.

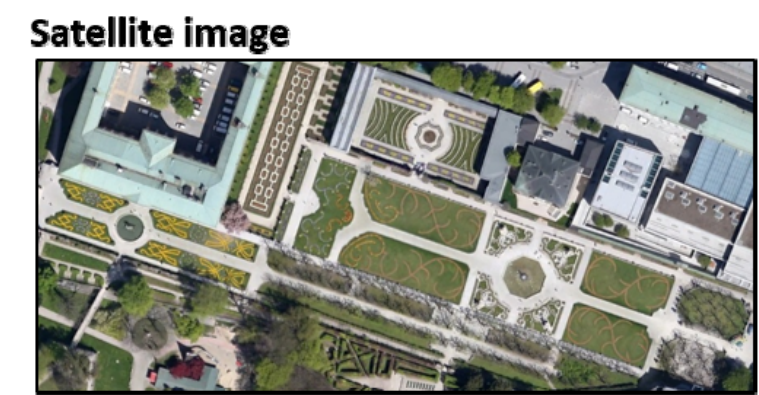

Composition of complex objects

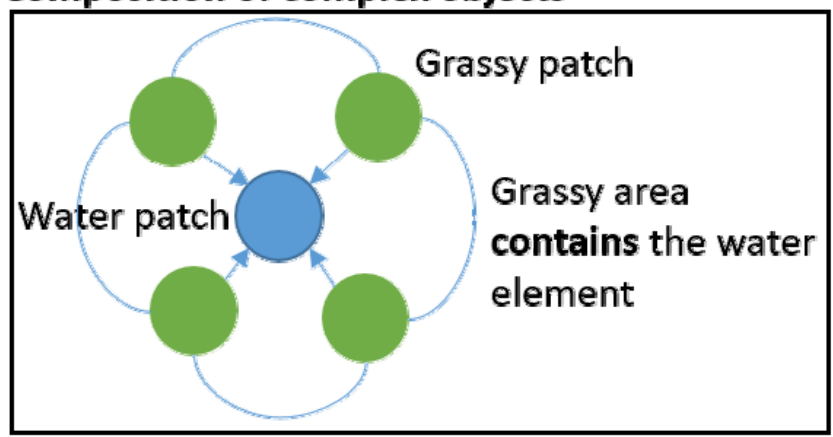

\section{Segmentation and Classification}

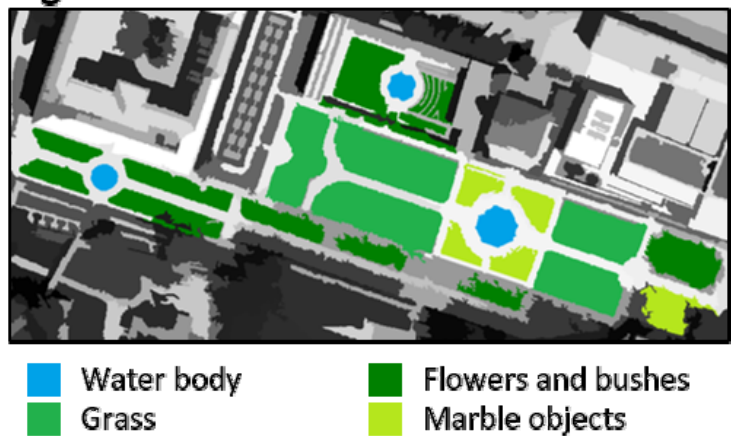

\section{Definition of places}

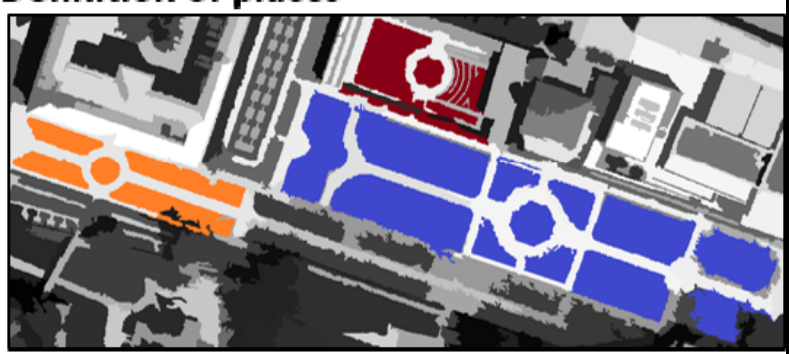

Water gardens

Complex object semantics from simple object properties and associations

Figure 1. Composition of water gardens

\section{CONCLUSION AND OUTLOOK}

OBIA has been proven to be useful to model 'vague' information or latent phenomena (Lang et al., 2014). While earlier OBIA approaches predominantly addressed physical environments, e.g. landscapes, the utilization of the underlying fuzzy methods for vaguely defined may appear obvious. Burnett and Blaschke (2003) stated that the landscape, especially in natural areas, is structured into continuously varying patterns that often defy crisp boundary placement at a fixed scale. Castilla and Hay (2008) point out that the width of the transition zone between two neighbouring landscape settings for which we have a name (e.g., 'forest' and 'sparse woodland').

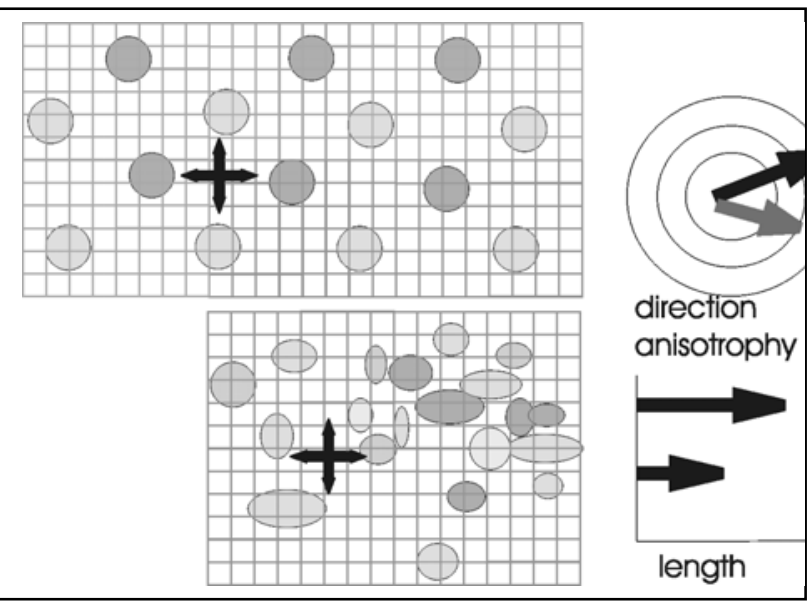

Figure 2: when dealing with objects instead of pixels information about size, range of size, shape, mean distance between objects, minimum distance, maximum distance, directionality and many more parameters can be used to describe their characteristics in addition to the spectral information (from Blaschke, 2003). While this figure was originally intended to address physical entities it may illustrate the potential of the concept to also be used for the representation of place 
In a joint effort, the OBIA and the GIScience research groups at the University of Salzburg will utilize and extend OBIA methods and methodologies to depict place. We strongly believe belief in the potential of the fuzzy membership classification of objects, sub-objects and super-objects. The fuzzy logic that forms the formal basis for classification assigns a membership value to each object between zero (totally ambiguous) and one (unambiguous) for each rule - and there can be hundreds of rules concatenated trough spatial, contextual, logic, hierarchical or any other parameter. This concept shall be further developed to one day implement the object-not-object dichotomy view as Burnett and Blaschke (2002) sketched out in an essayistic way.

GIScience increasingly acknowledges the importance of place research. In remote sensing, we may diagnose the apparent lack of methods, methodologies and a research framework, which would provide the basis to address vagueness in general and place-based perspectives in particular.

\section{REFERENCES}

Andrew, U., 1991. Qualitative spatial reasoning about cardinal directions. In Proc. of the 7th Austrian Conf. on Artificial Intelligence. Baltimore: Morgan Kaufmann, pp. 167.

Blaschke, T., 2003. Object-based contextual image classification built on image segmentation. In: IEEE (ed.) Advances in Techniques for Analysis of Remotely Sensed Data, pp. 113- 119.

Blaschke, T., 2010. Object based image analysis for remote sensing. ISPRS International Journal of Photogrammetry and Remote Sensing, 65(1), 2-16.

Blaschke, T., Donert, K., Gossette, F, Kienberger, S., Marani, M., Qureshi, S., Tiede, D., 2012. Virtual Globes: Serving Science and Society. Information, 3, 372-390.

Blaschke, T., G.J. Hay, M. Kelly, S. Lang, P. Hofmann, E. Addink, R. Feitosa, F. van der Meer, H. van der Werff, F. Van Coillie, D. Tiede, 2014. Geographic Object-based Image Analysis: a new paradigm in Remote Sensing and Geographic Information Science. ISPRS International Journal of Photogrammetry and Remote Sensing, 87(1), 180-191.

Burnett, C. and Blaschke, T., 2002. Objects / not-objects and near-decomposability: ecosystems and GI. In: NCGIA (ed.), GIScience 2002, Boulder, pp. 225-229.

Castilla, G., \& Hay, G. J., 2008. Image objects and geographic objects. In: Object-based image analysis. Springer Berlin Heidelberg. pp. 91-110.

Couclelis, H., 1992. People manipulate objects (but cultivate fields): beyond the raster-vector debate in GIS. In Theories and methods of spatio-temporal reasoning in geographic space. Springer, pp. 65-77.

Curry, M. R., 1996. The work in the world: geographical practice and the written word. Univ. of Minnesota Press.

Egenhofer, M.J. and Franzosa, R.D., 1991. Point-set topological spatial relations. International Journal of Geographical Information System, 5(2), pp.161-174.
Gehl, J., 1987. Life Between Buildings: Using Public Space, translated by Jo Koch. New York, NY: Van Nostrand Reinhold.

Gehl, J., 2010. Cities for People. Washington, DC: Island Press.

Gehl, J. and Svarre, B., 2013. How to Study Public Life. Washington, DC: Island Press.

Lang, S., Kienberger, S., Tiede, D., Hagenlocher, M., Pernkopf, L., 2014. Geons-domain-specific regionalization of space. Cartography and Geographic Information Science, 41(3), 214226.

Relph, E., 1976. Place and placelessness, vol. 67. Pion, London.

Tuan, Y.-F., 1977. Space and place: The perspective of experience. Univ. of Minnesota Press. 